S. I. Othman* and V. Anandam*, Department of Mathematics, College of Science, King Saud University, P.O. Box 2455, Riyadh - 11451, Saudi Arabia. e-mail: sadoon@ksu.edu.sa and vanandam@ksu.edu.sa

\title{
SINGULARITIES OF BOUNDED HARMONIC FUNCTIONS
}

\begin{abstract}
In a harmonic space, the property that $k$ is a compact set of removable singularities for bounded harmonic functions defined in a neighborhood of $k$ is independent of the neighborhood chosen.
\end{abstract}

\section{Introduction}

Let $k$ be compact and $w$ be open such that $k \subset w \subset \mathbb{R}^{n}(n \geq 2)$. Suppose that any bounded harmonic function in $w \backslash k$ extends as a harmonic function in $w$. Then, it can be shown (Theorem 9.9, Axler et al [4]) that for any open set $w_{0} \supset k$, if $u$ is bounded harmonic in $w_{0} \backslash k, u$ extends harmonically in $w_{0}$. Using a slightly different method, it is proved here that this result is true in Riemannian manifolds and in Riemann surfaces also; actually we work in the context of the Brelot axiomatic potential theory in harmonic spaces and mention some of its consequences.

\section{Preliminaries}

Let $\Omega$ be a connected and locally compact space which is not compact, provided with a sheaf of harmonic functions satisfying the 3 axioms of Brelot [5]. We assume that the constants are harmonic in $\Omega$. There may or may not be any potential $>0$ in $\Omega$. In case there is no potential $>0$ in $\Omega$, we fix an unbounded harmonic function $H \geq 0$ outside a compact set (Théorème 1.17 [1]); this function is the axiomatic analogue of $\log |x|$ in $\mathbb{R}^{2}$ and the Evans potential in a parabolic Riemann surface or in a parabolic Riemannian manifold (Nakai

\footnotetext{
Key Words: Harmonic extension, locally polar set, harmonic space

Mathematical Reviews subject classification: 31D05, 31C05

Received by the editors April 23, 1999

*The authors thank the Research Center, King Saud University for the grant Project No. (Math/1419/15).
} 
[7]). Then Lemma $1[2]$ and Theorem $2.2[3]$ together allow us to state the following useful assertion.

Lemma. In a harmonic space $\Omega$ with or without potentials $>0$, let $k$ be a compact set and $w$ be an open set such that $k \subset w$. Let $u$ be a harmonic function in $w \backslash k$. Then there exist a harmonic function $s$ in $\Omega \backslash k$ and a harmonic function $t$ in $w$ such that $u=s-t$ in $w \backslash k$. Moreover, $s$ can be assumed bounded near infinity if there are potentials $>0$ in $\Omega$; otherwise, for a suitable $\alpha, s-\alpha H$ is bounded near infinity.

\section{Nonremovable Singularities}

Recall (p.142 [1]) that a set e in a harmonic space is locally polar (resp. polar) in an open set $w \supset e$, if and only if there exists a superharmonic function (resp. a potential) $s$ in $w$ such that $s(x)=\infty$ on $e$; and a locally polar set $e$ in $w$ is polar if there exist potentials $>0$ in $w$ (p.47, Brelot [5]).

Theorem. In a harmonic space $\Omega$ with potentials $>0$, let $k$ be compact and $w$ be open such that $k \subset w$. Suppose there exists a bounded harmonic function in $w \backslash k$ which does not extend harmonically in $w$. Then for any open set $w_{0} \supset k$, there exists a bounded harmonic function in $w_{0} \backslash k$ which does not extend harmonically in $w_{0}$. The same is true in a harmonic space without positive potentials also, provided every point in $\Omega$ is locally polar.

Proof. Clearly it is enough to prove the theorem assuming $w_{0}=\Omega$. Note that $k$ is not locally polar in $w$ since there exists a bounded harmonic function $u$ in $w \backslash k$ which is not extendable as a harmonic function in $w$.

1) Assume that there are potentials $>0$ in $\Omega$. In this case $k$ is not polar in $\Omega$ (p.47, Brelot [5]). Let $h=\hat{R}_{1}^{k}$ in $\Omega$. Recall that

$$
R_{1}^{k}=\inf \{v: v \text { superharmonic } \geq 0 \text { in } \Omega \text { and } v \geq 1 \text { on } k\}
$$

and $\hat{R}_{1}^{k}$ is its lower semicontinuous regularization so that $h$ is a bounded harmonic function in $\Omega \backslash k$ which does not extend harmonically to $\Omega$.

2 ) Suppose now that there are no potentials $>0$ in $\Omega$. Since every point in $\Omega$ is locally polar in this case by the assumption, $k$ should contain at least two points $x_{i}(i=1,2)$ such that $u$ does not extend harmonically to any neighborhood of $x_{i}$. Consequently we can find two compact sets $k_{i}$ which are not locally polar and two open sets $w_{i}$ such that $k \supset k_{1} \cup k_{2}, k_{i} \subset w_{i} \subset \bar{w}_{i} \subset w$ and $w_{1} \cap w_{2}=\emptyset$. Let $u_{i}=\left(\hat{R}_{1}^{k_{i}}\right)_{w}$ in $w$ where the suffix $w$ indicates that the infimum is with respect to the functions defined in $w$, so that $u_{i}$ is a positive 
superharmonic function in $w$, harmonic in $w \backslash k_{i} \supset w \backslash k$ but not harmonic in the whole $w$.

Then using the lemma above, write $u_{i}=s_{i}-t_{i}$ in $w \backslash k$ where $s_{i}$ is harmonic in $\Omega \backslash k, t_{i}$ is harmonic in $w$, and $\left(s_{i}-\alpha_{i} H\right)$ is bounded near infinity. Here $\alpha_{i} \neq 0$. For otherwise, define

$$
v_{i}= \begin{cases}s_{i} & \text { in } \Omega \backslash k \\ u_{i}+t_{i} & \text { in } w .\end{cases}
$$

Then $v_{i}$ is a nonharmonic bounded superharmonic function in $\Omega$, a contradiction.

Let $s=\alpha_{2} s_{1}-\alpha_{1} s_{2}$ in $\Omega \backslash k ; s$ is harmonic and bounded near infinity. In $w \backslash k, s=\left(\alpha_{2} u_{1}-\alpha_{1} u_{2}\right)+\left(\alpha_{2} t_{1}-\alpha_{1} t_{2}\right)$ and hence it is bounded and harmonic in $w \backslash k$. Thus, $s$ is bounded harmonic in $\Omega \backslash k$, but $s$ cannot be extended harmonically in $\Omega$. For suppose $s$ extends harmonically in $w$; this in particular would imply that $s$ extends harmonically in $w_{1}$ and consequently (since $u_{2}, t_{1}$ and $t_{2}$ are all harmonic in $\left.w_{1}\right) u_{1}=\left(\hat{R}_{1}^{k_{1}}\right)_{w}$ is harmonic in $w_{1}$ and hence in $w$, a contradiction.

Remark. On the necessity of requiring in the above theorem that every point in $\Omega$ should be locally polar if $\Omega$ has no potentials $>0$ : Suppose $\Omega$ is a harmonic space without positive potentials and $k$ is a compact set as in the theorem. Then it may happen that $k$ reduces to a single point which is not locally polar. This necessitates the consideration of two different possibilities.

1) $k=\left\{x_{0}\right\}$ and $\Omega \backslash k$ is not connected. In this case there is no problem proving the above theorem; for, define a harmonic function $h$ in $\Omega \backslash k$, equal to 1 in one component and equal to 2 in the other components of $\Omega \backslash k$. Then $h$ is bounded and harmonic to $\Omega \backslash k$ which does not extend harmonically to $\Omega$. For an example of such a possibility, let $\Omega=\mathbb{R}$ with the affine functions as harmonic and take $k=\{0\}$.

2) On the other hand, if $k=\left\{x_{0}\right\}$ and $\Omega \backslash k$ is connected, the above theorem may fail; for, it may happen that there exists a bounded harmonic function in $w \backslash k$ which does not extend harmonically to $w$ whereas every bounded harmonic function in $\Omega \backslash k$ extends harmonically to $\Omega$. For an example of such a possibility, let $\Omega=[0, \infty)$ and define $h$ harmonic in $(a, b), a>0$, if it is affine and in $[0, c)$ if it is constant. Let $k=\{0\}$. Then, given any open set $w=[0, c), c<\infty$ we can find bounded harmonic functions in $(0, c)$ which do not extend harmonically in $w=[0, c)$; but any bounded harmonic function in $(0, \infty)$ being constant, every bounded harmonic function in $\Omega \backslash k$ extends harmonically to $\Omega$. 
Converse. Let e be a closed set in a harmonic space $\Omega$. We know that if $e$ is locally polar in $\Omega$, then $e^{0}=\phi$ and for any open set $w$, if $h$ is bounded harmonic in $w \backslash e, h$ extends harmonically to $w$. Let us propose its converse in the form: Let $e$ be a closed set contained in an open set $w, e^{0}=\phi$; if every bounded harmonic function in $w \backslash e$ extends harmonically to $w$, then e is locally polar in $\Omega$.

In this form the converse is true if there are potentials $>0$ in $\Omega$ (see 6.2.16, p.149 Constantinescu and Cornea [6]). For, if e is not locally polar, we can find a compact set $k \subset e$ which is not locally polar, since $\Omega$ is $\sigma$-compact. Then, $\left(\hat{R}_{1}^{k}\right)$ is a bounded harmonic function in $w \backslash e \subset w \backslash k$ which does not extend harmonically to $w$, a contradiction. (Note that the condition $e^{0}=\phi$ is necessary. For, consider the example of $\Omega=(0, \infty)$ with harmonic functions as locally affine functions; take $e=(0,1]$. Then any bounded harmonic function in $\Omega \backslash e$ being a constant extends harmonically to $\Omega$. But $e$ is not locally polar; however, if $e$ is compact, the condition $e^{0}=\phi$ is redundant.)

But, as the above remark shows, this converse need not be true if $\Omega$ does not have potentials $>0$ in $\Omega$. However, if we assume that each point is locally polar when there is no potential $>0$ in $\Omega$ (as in parabolic Riemann surfaces and in parabolic Riemannian manifolds), the above theorem shows that this converse is valid. For, if $e$ is not locally polar, let us choose a compact set $k \subset e$ which is not locally polar. Let $w_{0}$ be a relatively compact domain in $\Omega$ containing $k$; then $\left(\hat{R}_{1}^{k}\right)_{w_{0}}$ is a potential $>0$ in $w_{0}$. Now $\left(\hat{R}_{1}^{k}\right)_{w_{0}}$ is bounded and harmonic in $w_{0} \backslash k$ which does not extend harmonically to $w_{0}$; hence by the above theorem there exists a bounded harmonic function (in $w \backslash k$ and hence) in $w \backslash e$ which does not extend harmonically to $w$, a contradiction.

In particular, if $R$ is a Riemann surface or a Riemannian manifold of dimension $\geq 2$, hyperbolic or parabolic, and if $e$ is a closed set in $R, e^{0}=\phi$, then $e$ is locally polar in $R$ if and only if every bounded harmonic function in $R \backslash e$ extends harmonically in $R$.

Proposition. Let $\Omega$ be a harmonic space without potentials $>0$ in $\Omega$ where each point is locally polar. Let e be a closed set in $\Omega, e^{0}=\phi$. There exists a nonconstant positive harmonic function in $\Omega \backslash e$ if and only if there exists a nonconstant bounded harmonic function in $\Omega \backslash e$.

Proof. Let $s>0$ be a nonconstant harmonic function in $\Omega \backslash e$. Clearly $e$ is not locally polar since there are no potentials $>0$ in $\Omega$. Then as shown above, there exists a bounded harmonic function $u$ in $\Omega \backslash e$ which does not extend harmonically in $\Omega$. Clearly $u$ is a bounded nonconstant harmonic function in $\Omega \backslash e$. 
Remark. The assertion in the above proposition may not be valid in a harmonic space having potentials $>0$ (for example, $\Omega=\mathbb{R}^{3}$ and $e=\{0\}$ ); nor in a harmonic space where points are not necessarily locally polar.

\section{References}

[1] V. Anandam, Espaces harmoniques sans potentiel positif, Ann. Inst. Fourier, 22 (1972), 97-160.

[2] V. Anandam, Sur une propriétè de la fonction harmonique, Bull. Sc. Math., 101 (1977), 255-263.

[3] V. Anandam and M. Al Gwaiz, Global representation of harmonic and biharmonic functions, Potential Analysis, 6 (1997), 207-214.

[4] S. Axler, P. Bourdon and W. Ramey, Harmonic function theory, SpringerVerlag, N.Y., 1992.

[5] M. Brelot, Axiomatique des fonctions harmoniques, Les Presses de l'Université, Montréal, 1965.

[6] C. Constantinescu and A. Cornea, Potential theory on harmonic spaces, Grundleheren, 158, Springer-Verlag, 1972.

[7] M. Nakai, On Evans kernel, Pacific J. Math., 22 (1967), 125-137. 
S. I. Othman AND V. ANANDAM 